\title{
Synthesis, characterization and applications of sodium alginate and polyvinyl pyrollidine based clay reinforced biopolymers nanocomposites
}

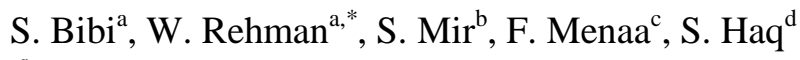 \\ ${ }^{a}$ Department of Chemistry, Hazara University Mansehra, KPK, Pakistan \\ ${ }^{b}$ Department of Chemistry, COMSATS University Islamabad, Abbottabad campus \\ KPK, Pakistan \\ ${ }^{c}$ Co-pionneer of Spectro-Fluor TM (aka Carbone-Fluorine Spectroscopy, \\ Fluorotronics USA, Inc. San Diego, CA, USA \\ ${ }^{d}$ Department of Chemistry, University of Azad Jammu and Kashmir, \\ Muzaffarabad, 13100 Pakistan
}

\begin{abstract}
Sodium alginate and PVP clay reinforced nanocomposites films were prepared by applying solvent casting technique using aqueous solution. The chemical behaviour and external morphology of nanocomposites films were studied by TGA, XRD, SEM, EDX and EDX Mapping technique. The pharmaceutical study was carried out by swelling study, erosion study. Ex-vivo permeate on study and dissolution study. The TGA study revealed the high miscibility between sodium alginate and PVP blends. The addition of MMT in blends convert crude surface of the films into interlinked porous state. The thermal stability, crystallinity, tensile and mechanical strength of blends has also increased by addition of MMT and drug.EDX and EDX mapping technology confirmed the consistency in mixing components and their equivalent distribution. The swelling and erosion attitude of films increases in the presence of PVP. The results of in vitro permeation study via rat skin and dissolution study showed the successful application of drug loaded formulations for sustained and targeted drug delivery. The different rate of drug release from formulation at $\mathrm{pH} 1.2, \mathrm{pH} 4.5$ and $\mathrm{pH} 6.8$ showed that the drug release is directed by PH. The sodium alginate and pvp based biopolymer nanocomposites films would be able for continual discharge of drug ceftriaxone.
\end{abstract}

(Received September 24, 2021; Accepted January 12, 2022)

Keywords: Sodium alginate, PVP, Nanocomposites, Ceftriaxone, MMT

\section{Introduction}

The class of polymers which have biocompatibility and biodegradability and are produced by natural system like plants, animals and microorganisms are named as biopolymers. They are manufactured by chemicals but their starting materials are organic and natural like amino acids, sugars etc the polymers having only c-c skeleton tried to combat decrepitude but hetero-atomic polymers are highly biodegradable. A sensible accumulation of chemical associations like anhydride, ester, and amide bonds produces biodegradability in polymers. The most common method of dilapidation is either by hydrolysis or breakdown of labile heteroatom bonds by enzymes, the active heteroatom bonds are mostly decayed by hydrolysis or enzyme action which produces scission in the main structure of polymer. This latest period has given the striking attention to biodegradable products in the different fields like wrapping, covering, casing, farming, cultivation, medication, tissue engineering, genetic material treatments, abrasion remedial, drug liberation systems and many others. Therefore the investigators are spending time to transform the conventional products into environment friendly products and in production of polymer nanocomposites from naturally present substances. Biodegradable polymers have produced the alertness amid pharmaceutical scientist as they not only improve the bioavailability of a drug but

\footnotetext{
* Corresponding author: sono_waj@yahoo.com https://doi.org/10.15251/JOBM2022.1411
} 
are also biocompatible. The biodegradable polymers are beneficent as their decay takes place inside the body without producing any foreign particles or residue.

In the last decades biodegradable polymers are functional independently or in blending with other polymers for drug transport means. A wide range of polymeric nanoparticles are prepared and investigated to advance drugs release capability and to lower their lethal possessions. The hybridization of natural polymers with synthetic results formation of materials possessing brilliant potentials especially high biodegradability, compatibility for biomedical functions like speedy identification by the immune system, and their elevated competence to transport drugs to the targeted cells. In literature biopolymer blends of natural and synthetic polymers have been reported as poly( $\mathrm{N}$-vinyl-2-pyrrolidone)-iota-carrageenan ( $\mathrm{PVP}=\mathrm{IC})$ [1], poly(N-vinyl-2pyrrolidone)-kappa-carrageenan $(\mathrm{PVP}=\mathrm{KC})$, poly(ethyleneoxide)-hydroxypropyl methylcellulose (PEO=HPMC) [2], poly(vinyl alcohol)-chitosan (PVA=C) [3].

Sodium alginate is natural polysaccharide extracted from marine brown algae (Phaeophyceae) [4]. Its structure is rectilinear, extended with negative charge and copolymer of 1,4-linked b-D-mannonic acid and a-L-gulonic acid [5]. Some bacteria like Pseudomonads azotobacter vinelandii also produce sodium alginate in abundance [6] It is a polyelectrolyte and is mostly valuable for sensing and regulation of outer chemical sign. Its skeleton compliance showlsensitivity to $\mathrm{PH}$ and potency of the aqueous medium [7] it is harmless biodegradable, biocompatible polymer having high potential for chelating and chemical amendments. Sodium alginate is used for the synthesis of many biopolymer nanocomposites of venerated quality like tissue engineering [8], cell organization [9] and control liberation of drug [10]. However, sodium alginate is easily decayed by enzymes and infected by microorganisms. These shortcomings are triumph over by hybridizing or grafting with other polymers. New products of desired characters are prepared by polymeric blending. The high miscibility and intermolecular coordination of blended polymers have been reported in literature [11-13].

$\mathrm{N}$ - Vinyl-2- pyrollidone (N-VP) is water soluble, un-hazardous, unionized and monomeric material, its polymerization into PVP is started either by radical, photo or thermal irradiation [14].PVP is biodegradable and biocompatible and has potency of combine reversibly to a variety of molecules in solution. It shows outstanding biocompatibility and least toxicity for living tissues therefore has become good transporter for drugs whether hydrophobic or hydrophilic [15]. PVP hydrogels and blends are used in cosmetics, food, printing, inks, paper, textile, medicine and so many other fields due to high solubility, elevated attraction for a range of polymers and resins, biodegradability and compatibility. It has carbonyl group which can make hydrogen bond with the hydroxyl group of sodium alginate.

The intentions of this work was to produce sodium alginate -PVP biopolymer nanocomposites films using clay (MMT) as reinforcing materials by solvent casting methodology. Sodium ceftriaxone (drug) was loaded in these films to study their physical and pharmaceutical characters.

\section{Experimental Procedure}

\subsection{Materials}

Sodium alginate was purchased from international laboratory USA,PVP and $\mathrm{KH}_{2} \mathrm{PO}_{4}$ was supplied by Daejung (South Korea), MMT (clay), $\mathrm{CH}_{3} \mathrm{COOH}, \mathrm{KCl}(99 \%), \mathrm{NaOH}(99 \%)$ was taken from Sigma Aldrich ${ }^{\circledR}$, USA, glycerol was supplied by Riedel-de-Haen, NaOAc by Merck (Germany).Ceftriaxone sodium by Global Pharmaceuticals (Islamabad, Pakistan) and distilled water from Comsats University Abbottabad campus.

\subsection{Preparation of the Films}

Drug loaded polyblend nanocomposites were prepared by solvent casting technique because it provides flush thickness, allocation, maximum optical limpidness and very little mist. The first step was preparation of solutions of sodium alginate, PVP, drug and clay in suitable volume of distilled water individually. After that sodium alginate and PVP polymer solutions were blended together and heated continually for 30 minutes on hot plate. The constant stirring of 
blended mixture was carried out by mechanical stirrer. The MMT solution was added as reinforcement into the blended mixture and further heating and stirring was done for 15minutes.After 15minutes drug solution and few drops of glycerol were added with steady stirring. The entire mixture was stirred for 30min again to get clear solution at fixed temperature. Then, this pure and clear solution was cast into petridishes and absolute drying was achieved by keeping it in an oven for $24 \mathrm{hrs}$. Solid even plastic films were produced after complete drying. We prepared 14 formulations

\subsection{Characterization}

\subsubsection{TGA analysis}

The measurement of weight loss in sodium alginate/ pvp films was done by Mettler Tolledo TGA/DSC Star 1 system. All samples were tested using Alumina 70ul pan in nitrogen atmosphere at scanning rate of $10{ }^{\circ} \mathrm{C} / \mathrm{min}$. keeping temperature range of $25^{\circ} \mathrm{C}-600^{\circ} \mathrm{C}$.

\subsubsection{XRD analysis}

$X$-ray diffraction was applied to investigate the amorphous and crystalline attitude by Philip XPERT PRO 3040/60. 10-90 was kept for $2 \theta$ range for $x$-ray diffraction study.

\subsubsection{SEM analysis}

SEM (Scanning electron microscopy) was applied to determine morphology of films using (TESCAN MIRA3) operated at 0-20kV.samples were kept at copper holder by sticky carbon tape and a skinny gold layer was coated by using gold stammer.

\subsubsection{EDX analysis}

EDX examination was done by TESCAN MIRA3.the formulations were put on copper holder following gold coating and the instrument was operated at $0-20 \mathrm{kV}$. The results confer about clearness, purity and elemental combination of the formulations.

\subsubsection{Swelling test}

The swelling capacity was measured in PH 6.8 (phosphate buffer), $\mathrm{pH} 4.5$ (sodium acetate buffer) and $\mathrm{pH} 1.2$ ( $\mathrm{HCl}$ solution).a $30 \mathrm{mg}$ films was put in $10 \mathrm{ml}$ of buffer solution inside a petridishes and was allowed to swell for 5miutes.the swelling capacity was noted with time gap of $1,2,3,4$ and 5 minutes orderly. At every interlude films was taken out from buffer solution, dried out by tissue paper and was weighed to note swelling point of films. The following principle was practiced to compute swelling proportion at equilibrium.

$$
\text { Swelling ratio }(\mathrm{SR})=\frac{\mathrm{Ws}}{\mathrm{Wd}}
$$

For water content capacity following formula was applied

$$
\text { Water content }(\%)=\left[\mathrm{W}_{\mathrm{S}} \mathrm{Wd} / \mathrm{W}_{\mathrm{S}}\right] 100
$$

where, $\mathrm{Wd}=$ Dry weight of film $(30 \mathrm{mg}) ; \mathrm{W}_{\mathrm{S}}=$ weight of the swollen film. The experiment was performed in triplicates. Mean and Standard deviation value were recorded in the graph.

\subsubsection{Erosion test}

The waterlogged films were set aside in open air to dehydrate them after noting their swelling capacity. Every dry formulation was weighed individually at different time gaps until a stable value was attained. The experiment was implemented in triplicate and erosion ratio was deliberated by using the set formula [16].

$$
\mathrm{ES}(\%)=\left[\mathrm{W}_{\mathrm{O}} \mathrm{W}_{f} / \mathrm{W}_{\mathrm{O}}\right] 100
$$

where, $\mathrm{W}_{\mathrm{O}}=$ weight of swollen film, $\mathrm{W}_{f}$ final dry weight. 


\subsubsection{Calibration curve plot}

The standard calibration curve was contrived to obtain linear equation for ceftriaxone sodium. This equation is designed to conclude the drug concentration in all formulations.100mg of ceftriaxone sodium was dissolved in $100 \mathrm{ml}$ of $\mathrm{KH}_{2} \mathrm{PO}_{4^{-}} \mathrm{pH}$ 6.8.10 dilutions were put in order in the series of $2-20 \mathrm{ug} / \mathrm{ml} . \mathrm{KH}_{2} \mathrm{PO}_{4^{-}} \mathrm{pH} 6.8$ was used as blank for analysis of dilution UV/VIS spectrophotometer of at $323 \mathrm{~nm}[17]$.

\subsubsection{Ex-vivo permeation test}

The Franz Diffusion Cells were used to investigate the permeation analysis of ceftriaxone sodium via rat skin.

\subsubsection{Preparation of rat skin}

Sprague-Dawley rats were taken from department of pharmacy, COMSATS Abbottabad to execute permeation study. The rats were by chloroform. The rat skin was bald by hand and electric blade. Surgical blades and scissors were applied to get rid of belly skin and underlying fat. The skin was dipped in $0.9 \% \mathrm{NaCl}$ normal salt solution for $2 \mathrm{hrs}$ to wipe out leaching out dermal fragments and enzymes. The skin was thoroughly washed in water, dried out and enclosed inside aluminum foil to store in freezer till used. Prior to begin the experiment, the skin softening was carried out at room temperature [18].

\subsubsection{Set up of Franz diffusion cell}

The rat skin was placed between the centre of two compartment of Franz diffusion cell in such a manner that dermal part of skin was towards the receptor side and stratum corneum section was towards donor part. By using clips skin was fixed between two compartments of Franz cells. The receptor part was filled with $4.5 \mathrm{ml}$ of phosphate buffer of $\mathrm{pH}$ 6.8. These Franz cells were placed in water bath at fixed temperature of $37^{\circ} \mathrm{C} .40 \mathrm{mg}$ of drug loaded film was introduced in the donor part. A small magnetic stirrer was used to produce continual stirring in buffer solution inside the receptor section. A time gap of 1, 2, 3, 4, 5, 6 and 24 hrs was applied to remove almost $200 \mu \mathrm{l}$ fluid from receptor part and drug absorbance was noted by UV-Visible Spectrophotometer at 323 $\mathrm{nm}$. The removed buffer amount was maintained by adding buffer repetitively at every reading.

\subsubsection{Dissolution study}

The dissolution study was executed by dissolving $25 \mathrm{mg}$ of drug loaded films in $100 \mathrm{ml}$ of $\mathrm{KH}_{2} \mathrm{PO}_{4-}$ pH 6.8 at $37{ }^{\circ} \mathrm{C}$. After every $10 \mathrm{~min} 5 \mathrm{ml}$ of sample was taken out and level of buffer solution was maintained by adding buffer solution. The release of ceftriaxone was investigated by noting absorption using PBS of pH 6.8 as the reference standard at UV-VIS spectrophotometer at $323 \mathrm{~nm}$.

\section{Results and discussion}

Synthesis, characterization and applications of sodium alginate/pvp clay reinforced biopolymer a nanocomposite was carried out. Translucent, white, elastic and plastic like films were produced by solution casting method.

\subsection{TGA analysis}

The thermogravimetric analysis shows the strength of samples over variable temperature ranges. The Figure 1(A) showed TGA thermogram of formulations AVD100, AVD70 and AVD50. These formulations consist of different amount of polymers. These curves showed that different stages of degradation are observed for all three formulations in which the amount of nanoclay is constant but the amount of polymers vary from 100/0, 70/30, and 50/50 of sodium alginate and PVP respectively. The initial weight loss occurs at $120{ }^{\circ} \mathrm{C}$ and it is associated with water [19]. Table 1 showed that 23.9-18.4\% weight loss occurs at this temperature. AVD70 and 
AVD100 showed minimum and maximum water loss of about $13 \%$ and $23.9 \%$ respectively. Second weight loss is observed at $200{ }^{\circ} \mathrm{C}$ which involves a complex process of degradation of polysaccharide rings, it is expected main polymeric chains are broken in the $2^{\text {nd }}$ stage of degradation. [20]. Third stage was found at $334-435{ }^{\circ} \mathrm{C}$, which is related to the decomposition of PVP Chains [21].

At $400{ }^{\circ} \mathrm{C}$ formulations AVD100 and AVD70 showed maximum and minimum weight loss of about 73.7 and 68\%, respectively. The degradation of PVP takes place between 330-435 ${ }^{\circ} \mathrm{C}$. The increase in thermal stability of sodium alginate upon addition of PVP is due to formation of H-bonding among hydroxyl groups of sodium alginate and carbonyl group of PVP in SA/PVP blend [22].Table showed that at $600{ }^{\circ} \mathrm{C}$ maximum residue $24.3 \%$ was observed in formulation AVD70 and minimum residue $18.1 \%$ was observed in formulation AVD100. Formulation AVD100 contain high amount of sodium alginate while formulation AVD70 contain Sodium Alginate and PVP (70/30). Residue percentage showed that sodium alginate's thermal stability increases with addition of PVP.

Figure 1(B) showed TGA thermo gram of formulations AVC10 and AVC20. Table 1 showed that initial weight loss occurs at temperature $120{ }^{\circ} \mathrm{C}$ which is related to water. Second weight loss occurs at $200{ }^{\circ} \mathrm{C}$ due to depolymerization of polymers. At $400^{\circ} \mathrm{C} \mathrm{AVC} 10$ and AVC20 showed weight loss $77.6 \%$ and $61.1 \%$ respectively.AVC20 which have high concentration of clay is thermally more stable than AVC10 as clay improve thermal stability.

Figure 1(C) showed TGA thermogram of formulation AV50-AVD2. These formulations consist of different amount of drug and same amount of polymers. The initial weight loss occurs at $120^{\circ} \mathrm{C}$ which relates to the loss of water. Formulation AVD1.5 and AV50 showed minimum and maximum weight loss of about $13 \%$ and $23.8 \%$. Second weight loss occurs at $200{ }^{\circ} \mathrm{C}$. At this temperature formulation AVD1.5 showed minimum weight loss of about $23.5 \%$ and formulation AV50 showed maximum weight loss of about $42.5 \%$. Table also showed that at $400{ }^{\circ} \mathrm{C}$ AV50, AVD0.5, AVD50, AVD1.5 and AVD2 showed weight loss of 70.5, 69.5, 71.1, 64.1 and 65.1 respectively. At $600^{\circ} \mathrm{C}$ maximum residue of about 25.6 is given by AVD1.5 and AVD50 showed minimum residue of about $19.5 \%$ which indicate that AVD1.5 is thermally more stable.

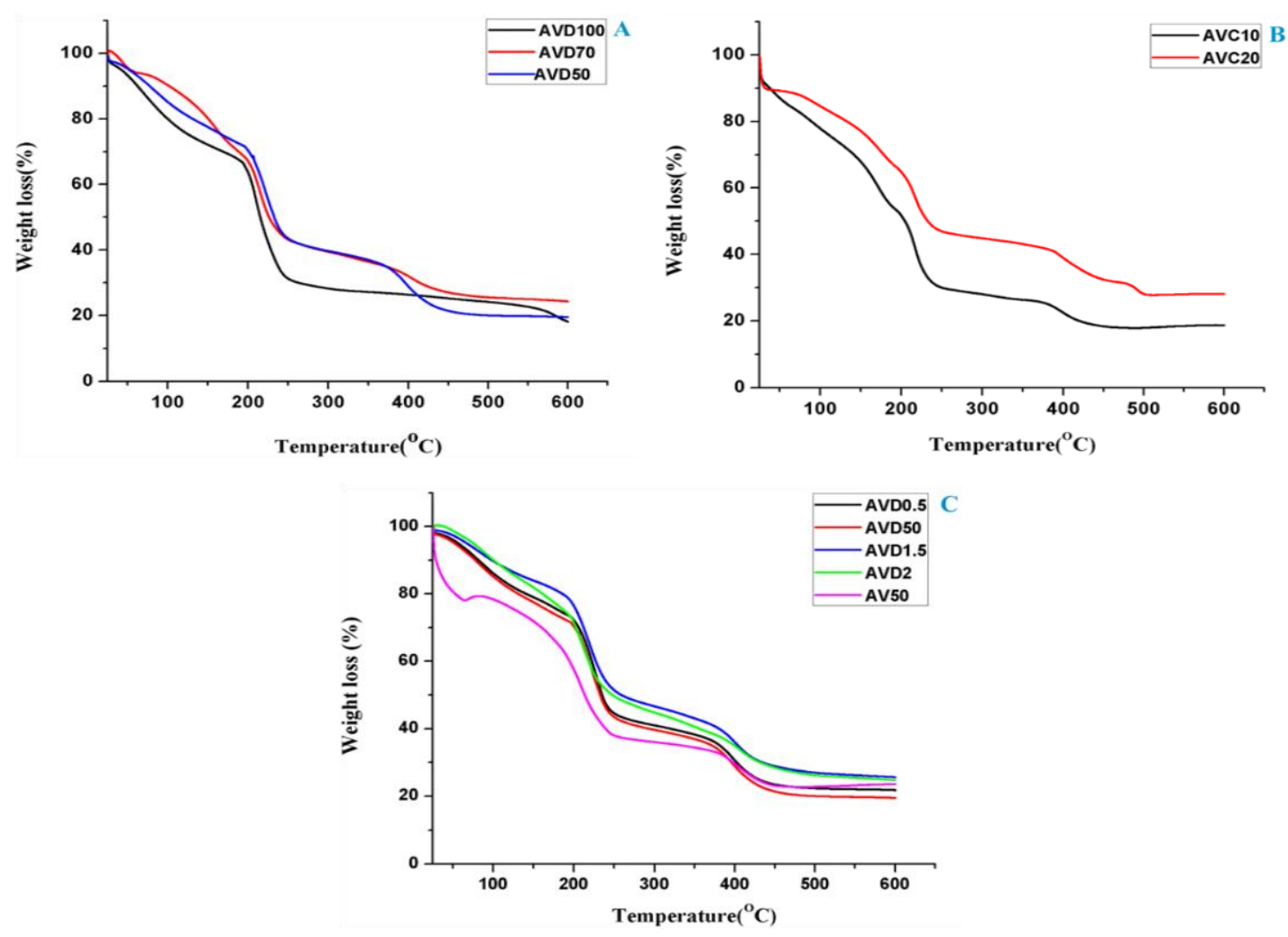

Fig. 1. TGA thermograms of drug loaded and non-drug loaded Sodium alginate /PVP clay reinforced nanocomposite films (A) AVD100-AVD50 (B) AVC10-AVC20 (C) AV50, AVD0.5-AVD2. 


\subsection{XRD analysis}

These formulations are consisted of two polymers (Sodium alginate and PVP), nanoclay (MMT) and drug (Ceftriaxone). According to previous studies Sodium alginate gives sharp peaks at $6.55^{\circ}, 6.49^{\circ}, 13.6^{\circ}, 14^{\circ}$ and $23^{\circ}$ [23] PVP appears at $2 \theta=13^{\circ}$ and $21^{\circ}[8,24-25]$. MMT appears at $6.98^{\circ}, 8.5^{\circ} \cdot 10.5,19.7^{\circ}, 20.3^{\circ}, 61.8^{\circ}, 21.3^{\circ} .35 .5^{\circ}$ An intense peak a between $2 \theta=5-6^{\circ}$ is seen in all the samples due to crystalline nature of MMT [26-28]. These peaks arise at lower plane instead of $2 \theta=7.48^{\circ}$ refers that during blending, the polymers are inserted and intercalated in between the spaces of layered silicates which spaced out the clay layers. All the samples of this series contain drug which is giving peaks between $11-13^{\circ}$.

The series AVD100-AVD30 in Figure 2 (A) all the samples produce broad peak at $2 \theta=22^{\circ}$ and $2 \theta=38^{\circ}$, which showed their amorphous nature of low crystalline value [28]. AVD70-AVD30 (Sodium alginate/PVP/Drug/MMT the polymer matrix has amorphous phase dominant over crystalline. Therefore, the crystalline manifestations related to the addition of MMT and Drug is not prominent in these polymer nanocomposites. In sample AVD70-AVD30, the PVP gives sharp peak at $2 \theta=13^{\circ}$ and $21^{\circ}[8,24-25]$ which is absent in AVD100 due to absence of PVP. Figure 2(B) gives XRD patterns of series AVC50 (6) -AVC20 where concentration of polymer is constant $50 / 50$ sodium alginate/PVP but cay is varying i.e. 6\%, 10\%, 20\% in AVC50, AVC10 and AVC20 respectively.

Mehndi et al., reported that MMT gives sharp peak at $2 \theta=8.5^{\circ}$ which shows highly crystalline behavior of clay (MMT).but this peak is shifted towards the lower $2 \theta$ value because of intercalation of polymer into clay sheets [29] and give broad peaks in the range of $2 \theta=5-6^{\circ}$.the characteristic reflection of MMT are not seen in this series(AVC50-AVC20) which indicates that MMT exfoliate after its interaction with polymer nanocomposites of sodium alginate and PVP having lower amount of clay(3\%).when loaded amount of MMT was raised to 6\%, $10 \%$ and $20 \%$ its diffraction peak move from 8.5 to $5-6^{\circ}$.this shows that polymers chains intercalate into the interlayer space of MMT [30] constituting an intercalated and exfoliated structure of nanocomposites at relatively higher concentration of MMT [31]. All samples showed peaks at 22 and $38^{\circ}$ inferring semi amorphous nature and decrease in crystallinity is due to addition of sodium alginate in PVP [28].

Here in Figure 2(c) XRD patterns of drug loaded nanocomposites are compared with one non-loaded nanocomposites to check the upshots of drug on the character of nanocomposites. The sample AV50 has 0\% drug whereas the amount of drug in sample AVD0.5.AVD50, AVD1.5, AVD2 is $10 \%, 15 \%, 30 \%$, and $40 \%$ respectively. The prior data showed that ceftriaxone has crystalline structure. Many sharp peaks are given by its pure form between $11-13^{\circ}, 18-25^{\circ}$ and $28^{\circ}$. All the samples except AD50 showed peak between $11-13^{\circ}$, and $18-27.8^{\circ}$ which confirms its presence and inferred that as the drug concentration is increased the crystallinity of nanocomposites is also enhanced. In some samples the drug peaks showed decrease in intensity as compared to pure sample which showed that drug has interacted with the polymers which results a change in atomic densities in a specific plane of crystal lattice [23].All samples showed sharp peaks at $5^{\circ}$ and $6^{\circ}$ of MMT. In accordance Mehndi et al. who reported that pure MMT give at $2 \theta=8.5^{\circ}$ confirming its crystallinity. But in nanocomposites polymers intercalate into clay sheets [29] and shifts the peak to lower and broad peaks in the range of 2 $\theta=5-6$ are seen. Similarly sodium alginate and PVP also produce broader peaks due to their amorphous nature [32]. Figure 2(B) gives XRD patterns of series AVC50 (6) -AVC20 where concentration of polymer is constant 50/50 sodium alginate/PVP but cay is varying i.e. 6\%, 10\%, 20\% in AVC50, AVC10 and AVC20 respectively.

Mehndi et al., reported that MMT gives sharp peak at $2 \theta=8.5^{\circ}$ which shows highly crystalline behavior of clay (MMT).but this peak is shifted towards the lower $2 \theta$ value because of intercalation of polymer into clay sheets [29] and give broad peaks in the range of $2 \theta=5-6^{\circ}$.the characteristic reflection of MMT are not seen in this series(AVC50-AVC20) which indicates that MMT exfoliate after its interaction with polymer nanocomposites of sodium alginate and PVP having lower amount of clay(3\%).when loaded amount of MMT was raised to 6\%, 10\% and 20\% its diffraction peak move from 8.5 to $5-6^{\circ}$.this shows that polymers chains intercalate into the 
interlayer space of MMT [30] constituting an intercalated and exfoliated structure of nanocomposites at relatively higher concentration of MMT [31]. All samples showed peaks at $22^{\circ}$ and $38^{\circ}$ inferring semi amorphous nature and decrease in crystallinity is due to addition of sodium alginate in PVP [28].
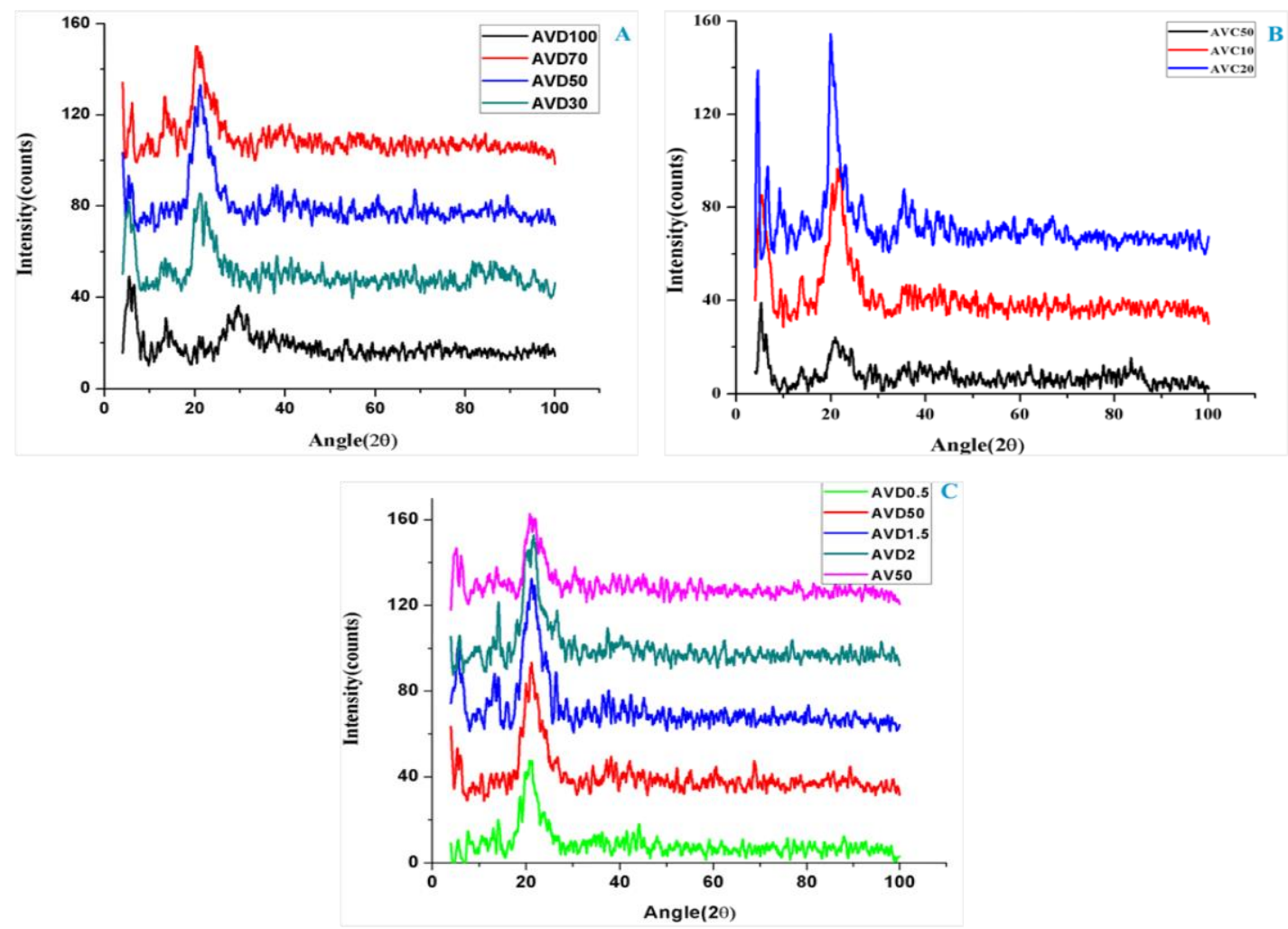

Fig. 2. XRD patterns of non-drug loaded and drug loaded Sodium Alginate/PVP/clay reinforced nanocomposite films (A) AVD100-AVD30 (B) AVC50(6)-AVC20(C)AVD50,AVD0.5-AVD2A,AV50.

\subsection{Scanning Electron Micrographs}

TO investigate the size of particles, morphology and compatibility among polymers, MMT and drug SEM images of three formulations AP50, APC10 and APD0.5 were taken. All the formulations contain sodium alginate/PVA/clay. The AP50 and APC10 are without drug while APD contain $10 \%$ drug. The pure sodium alginate revealed soft even and consistency in its morphology but when PVA is added for blends formation the morphology is converted from smooth to microphase separated which inferred the change in miscibility of two types of polymers from good to certain value of miscibility. This certain value of miscibility and good miscibility are essential for varying physical characters of materials. In these formulations the MMT (reinforcement) is consistently disseminated in the matrix of film. This proved its high compatibility in the polymer matrix, as there were no gaps and cracks in the formulations.

Figure 3(A) gives surface properties of AV50 (alginate/PVP/clay) in ratio of 50/50/3 respectively. A dark background represents the consistency in matrix while nanoclay is dispersed equivalently in entire matrix in the form of tiny chips. Figure 3(B) showed APC50 (alginate/PVP/clay) in ratio of 50/50/10 respectively. When the concentration of nanoclay is increased over here the non-dispersed clay particles caused agglomeration of clay in large sized integrated clay pack. Figure 3 (C\&D) APD0.5 showed (alginate/PVP/clay/drug) in ratio of 50/50/3/10 respectively. Addition of 10\% drug changed the morphology of nanocomposites. The drug appear in the form of large blocks while nanoclay made small dot like structures which are consistently dispersed in the formulation proving its high compatibility. Presence of drug in the form of large sized blocks confirmed its crystalline structure which is also revealed by previous 
studies [33-34]. Moreover, these images showed that all components are homogeneously dispersed in a polymer matrix.
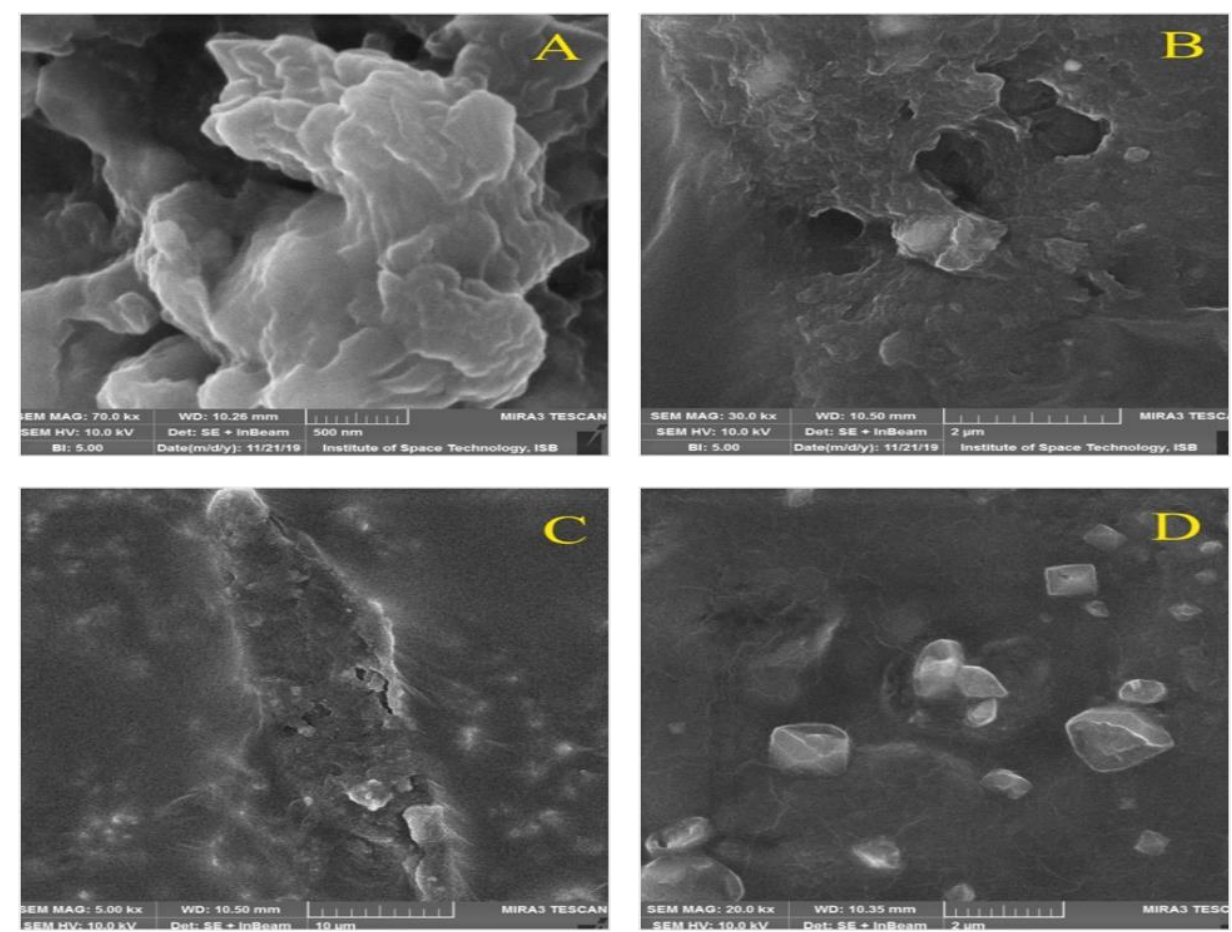

Fig. 3. Scanning Electron Micrographs Of sodium alginate/PVA/clay reinforced nanocomposites films (A) AV50 at x70, 000(B) AVC50at x30, 000 (C) AVD0.5 atx5, 000 and (D) AVD 0.5 at x20, 000magnifications.

\subsubsection{EDX analysis}

EDX analysis was carried out to check the purity and elemental composition of mixing component of AV50, AVC50 and AVD 0.5. Spectrum 14 represent the elemental composition of AV50.It has peaks of $\mathrm{C}, \mathrm{O}, \mathrm{Na} . \mathrm{Ca}, \mathrm{Mg}$, Si. The intense peak of C.O and $\mathrm{Na}$ shows that the polymer is in greater proportion i.e. Sodium alginate and PVP. While peaks of $\mathrm{Ca}, \mathrm{Mg}, \mathrm{Si}$ and $\mathrm{Na}$ having low intensity indicates the presence of MMT. There is no peak for impurity. AVC50 also showed the peaks for polymers and MMT. AVD0.5 contain drug too which was absent in above two spectrum that's why we can see the peak of sulphur confirming presence of ceftriaxone drug. 

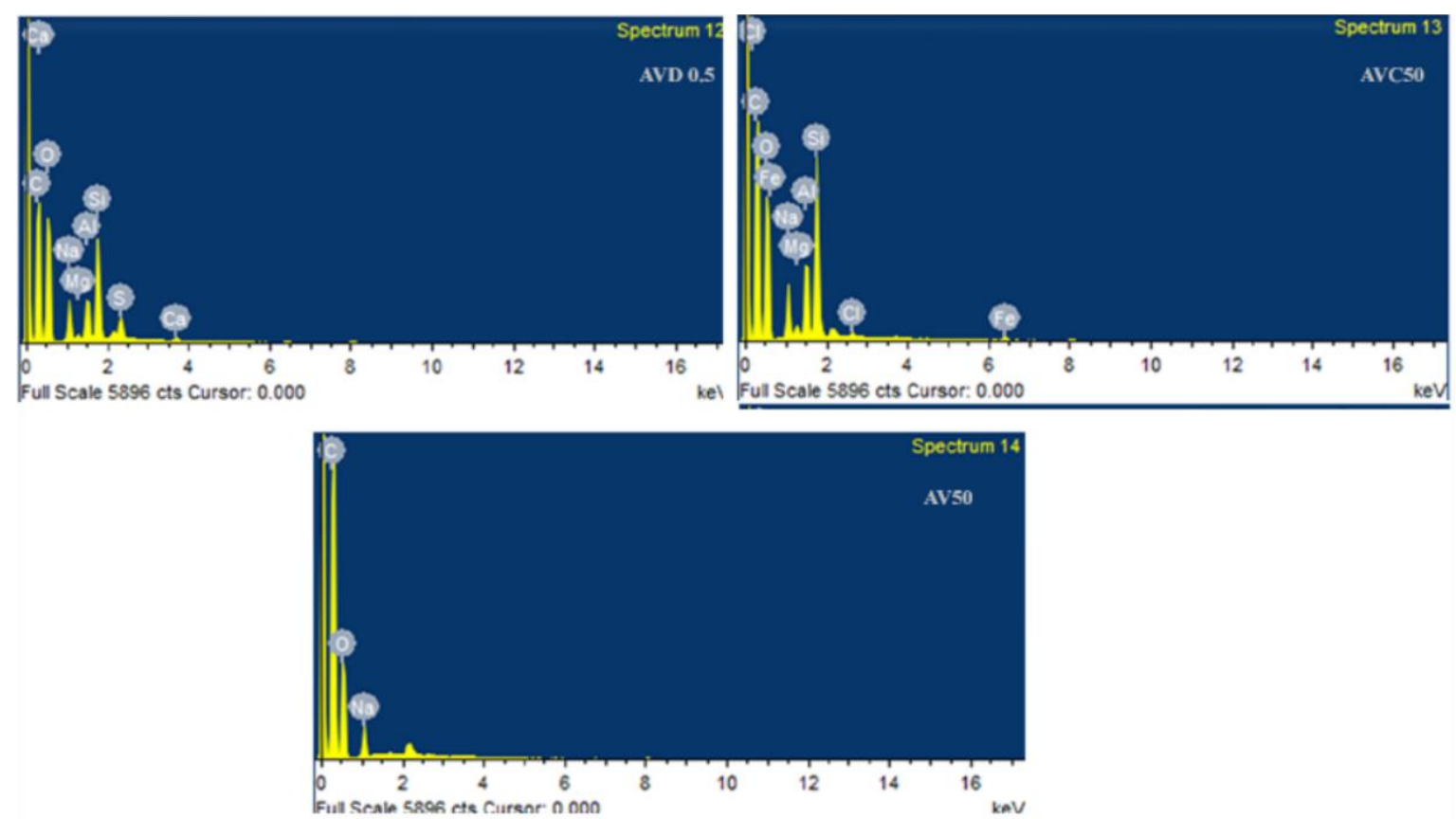

Fig. 4. EDX profile of formulations (A) AP50, (B) APC50 \& (C) APD 0.5 AV50.
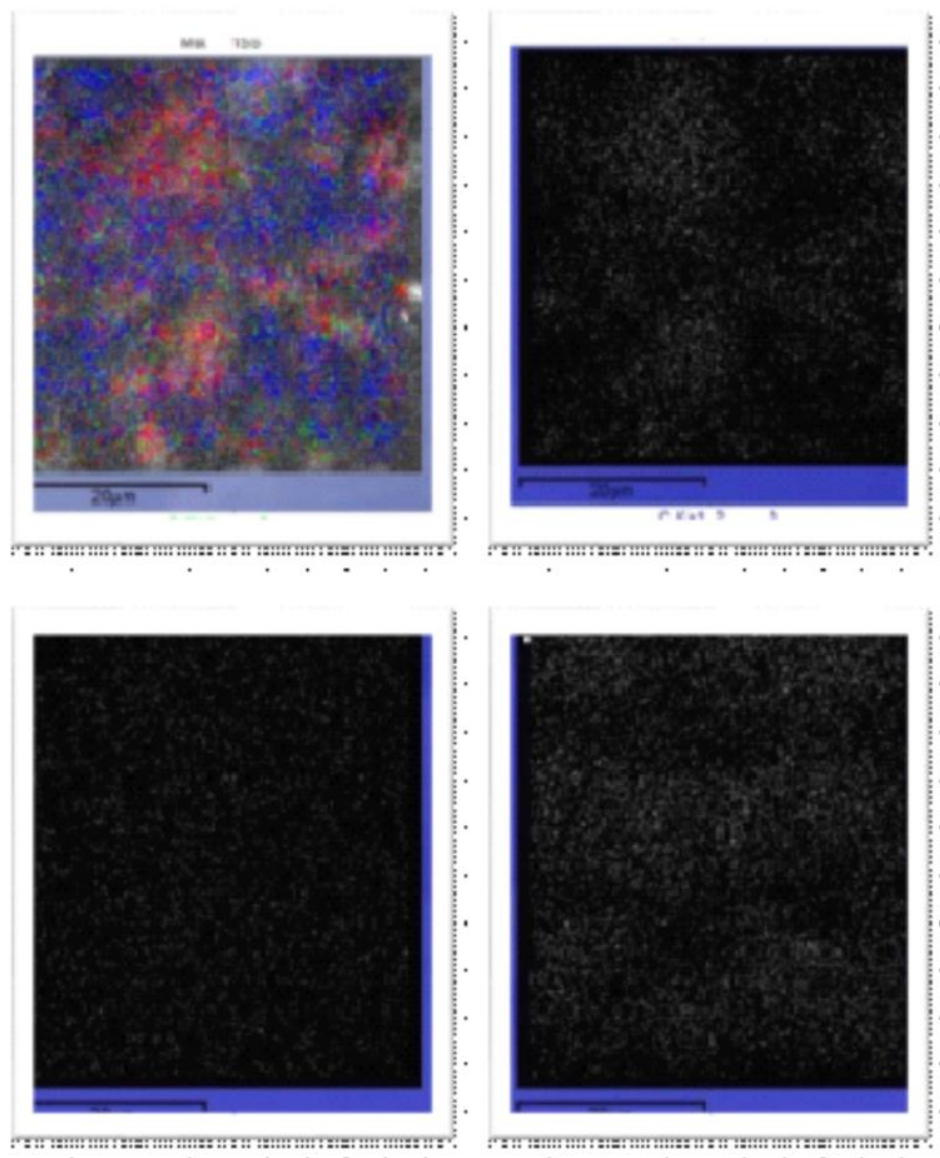

Fig. 5. Elemental composition of AV50. 


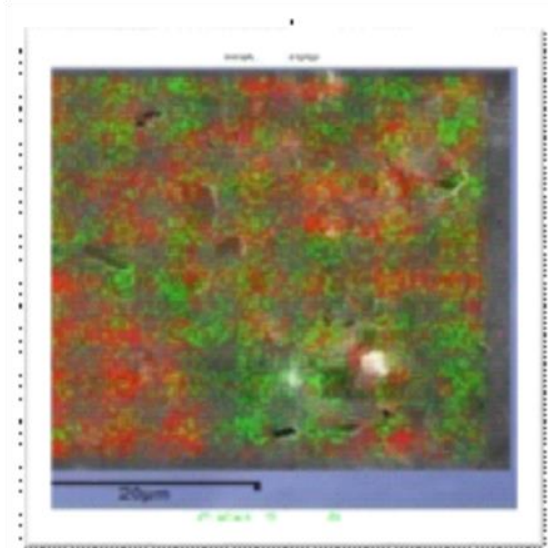

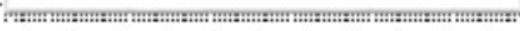

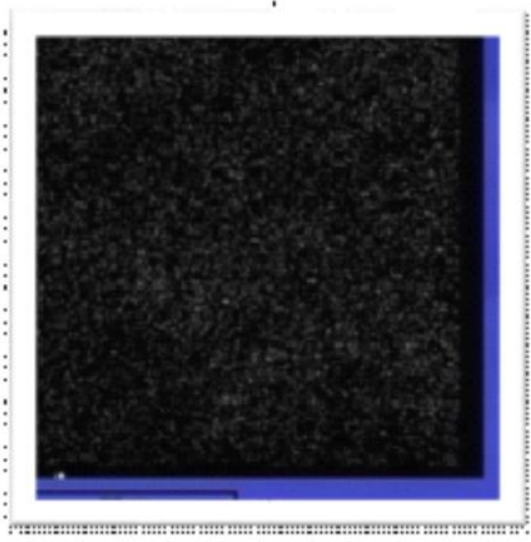

Fig. 6. Elemental composition of AVC50.

Electron Image 1
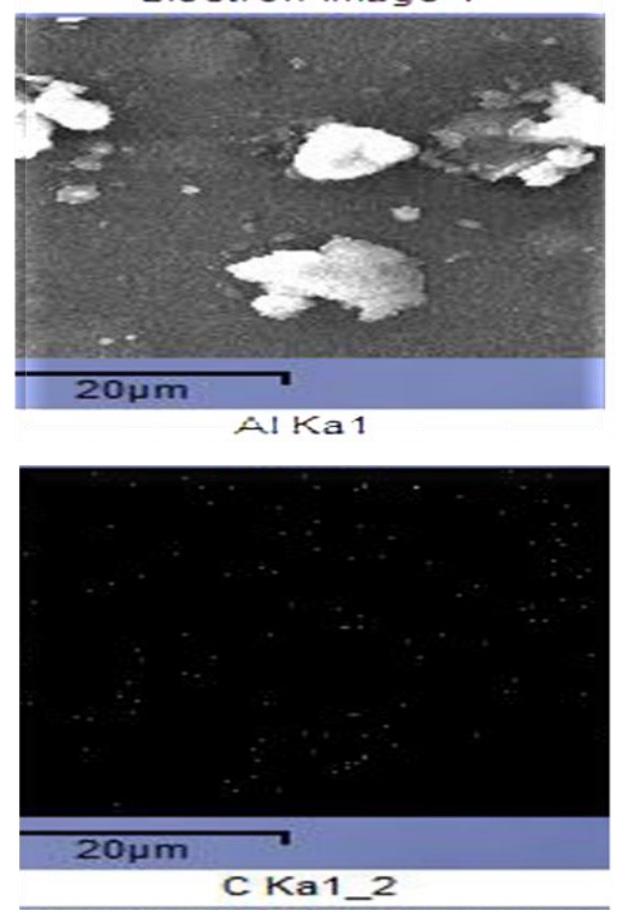

O Ka1
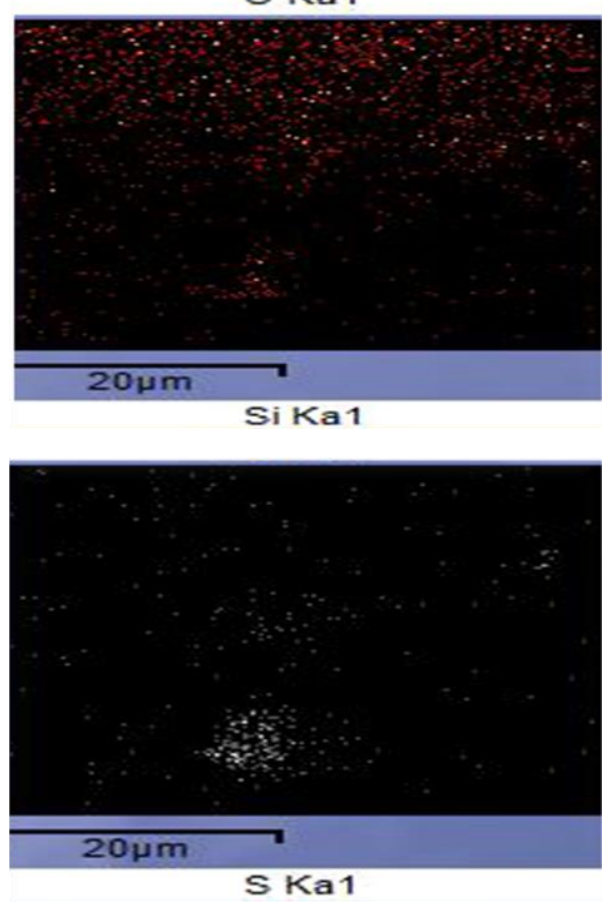

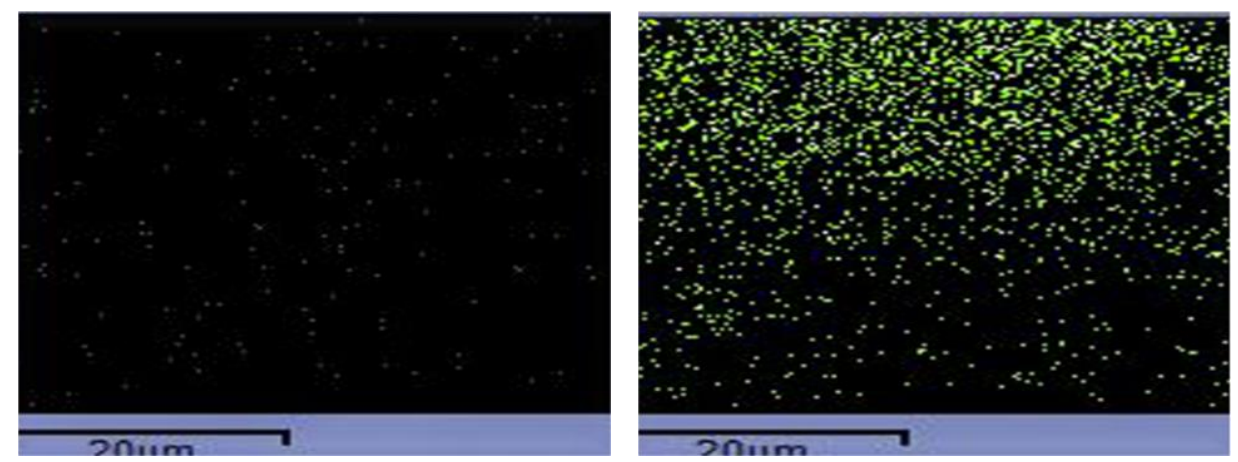

Fig. 7. Elemental composition of AVD 0.5.

\subsubsection{Swelling Study}

Figure 8(A) show the swelling behaviour of non-drug loaded sodium alginate/PVP/Clay reinforced nanocomposites films. Buffer solutions of $\mathrm{pH} 1.2,4.5$ and 6.8 were used to investigate the swelling behaviour. The buffers of $\mathrm{pH} 6.8,4.5$ and 1.2 were prepared to study the swelling action of nanocomposites film of composition Sodium alginate/PVP/Clay. Swelling action is controlled by the nature of polymer, extent of cross-linking and polymer solvent aptitude. The polymer nanocomposites showed variation in their swelling behaviour. The series AV100- AV30 is formulated with constant amount of clay but the varied amount of polymers. The formulation AV30 showed maximum swelling ratio containing 30\% sodium alginate and $70 \%$ PVP Figure 8(A) evaluated decrease in swelling with the increase in sodium alginate concentration in the prepared films. The least swelling is seen in AV100 which is made up of 0\% PVP and100\% sodium alginate. An increase in PVP concentration increased swelling due to hydrophilic behaviour of PVP [35] which has elevated the swelling aptitude [36-37] when we increase the quantity of PVP in Sodium alginate, it increase the number of carbonyl groups which have strong hydrophilic character and collaborate with water by $\mathrm{H}$-bonding resulting swelling increases [35]. In buffer of $\mathrm{pH} 6.8$ the maximum swelling action was seen as compared to 4.5 and 1.2 .These results evaluate an increase in swelling ratio with an increase in the $\mathrm{pH}$ of the buffers. High $\mathrm{pH}$ value causes dissociation of carboxylic acid groups which strengthen the electrostatic repulsive force among anions of carboxyl ate and .cause additional extension in polymers network consequently more swelling takes place [38].

In series AVC50 (6)-AVC20, the AVC20 having the maximum amount of clay showed least swelling capacity which inferred a decrease in swelling behaviour with an increase in MMT. This decrease in swelling ratio is due to the formation of hydrogen bonding network of MMT with the polymers which enhances the surface coarseness of films and closed the water penetration corridor resulting decrease in water compassion of the [39]. Secondly the Si-O-Si group of MMT is the basic functional group which is responsible for communication to drug and polymer. An increase in the clay concentration produce more $\mathrm{Si}-\mathrm{O}-\mathrm{Si}$ groups for interaction and creates more cross linking in the structure which increases the number of pores but decrease the pore size and this cause decreases in the swelling ratio [23]. Thirdly MMT is less hydrophilic than sodium alginate and PVP so its merger lowered the swelling ratio. 

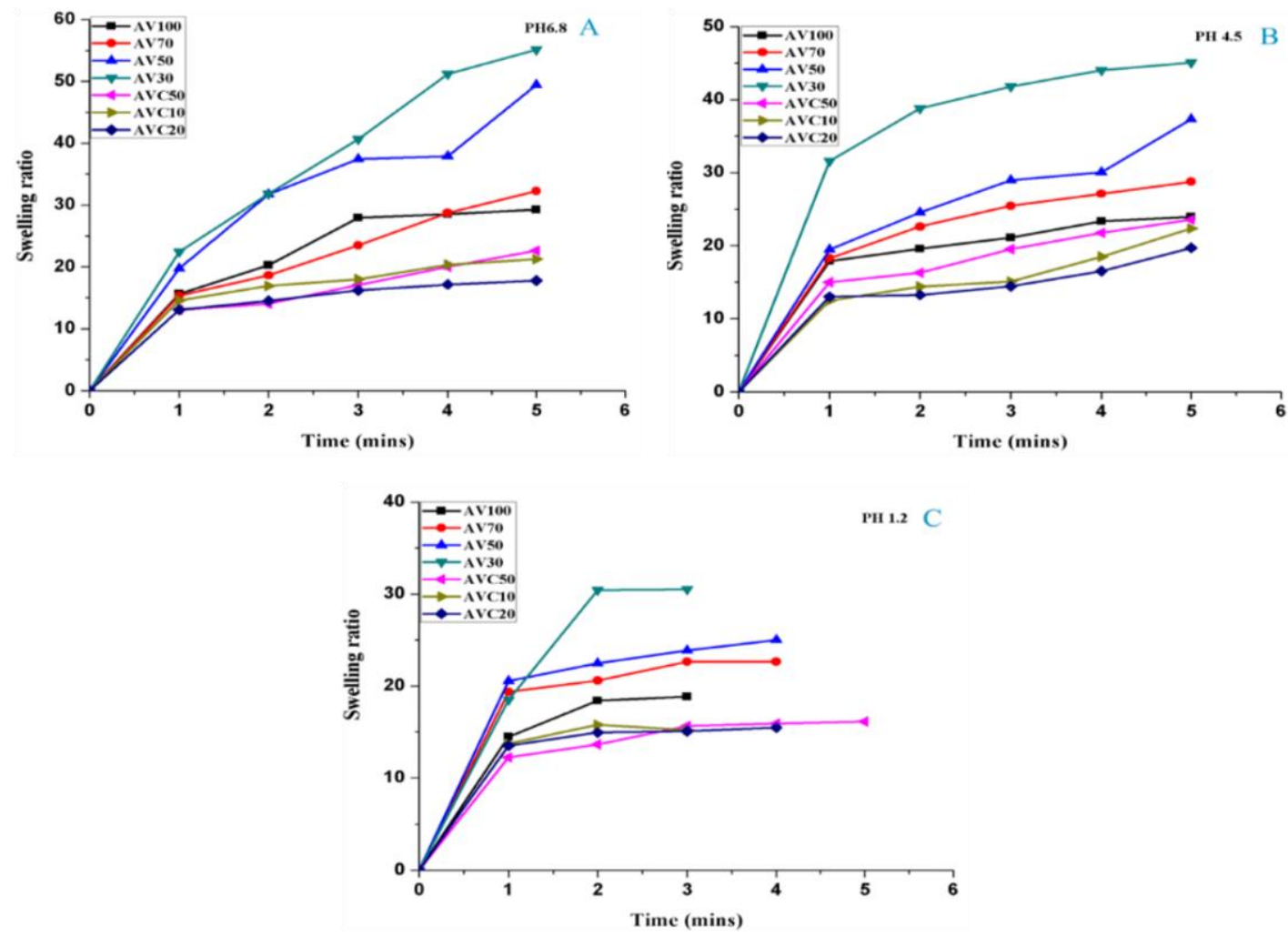

Fig. 8. Swelling ratio of non-drug loaded Sodium alginate/PVP/Clay nanocomposite films in (A) Phosphate- $\mathrm{pH} 6.8$ buffer solutions (B) NaOAc-pH 4.5 and (C) $\mathrm{HCl}-\mathrm{pH} \mathrm{1.2.}$

The Figure 9(A) shows series AVD100- AVD30 containing a constant amount of clay and drug but the varied amount of polymers. Swelling ratio increases with the increase in PVP concentration or decrease in sodium alginate concentration. Maximum swelling ratio is seen in formulation AVD30 that have 30\% sodium alginate and 70\% PVP in phosphate buffer of $\mathrm{pH} 6.8$. The Figure 9(A) shows that the decrease in the concentration of PVP in formulation AVD50 (50\%sodium alginate and 50\% PVP) decrease the swelling ratio. Additional decrease is seen in AVD70 (sodium alginate 70\% and PVP 30\%) and AVD100 (sodium alginate 100\% and PVP 0\%). AVD100 showed least swelling ratio that have100\% sodium alginate and PVP 0\%. An increase in PVP concentration increased swelling due to hydrophilic behaviour of PVP [35] which has elevated the swelling aptitude [38]. When we increase the quantity of PVP in Sodium alginate, it increase the number of carbonyl groups which have strong hydrophilic character and collaborate with water by H-bonding resulting swelling increases [35].

In buffer of pH 6.8 the maximum swelling action was seen as compared to 4.5 and 1.2. These results evaluate an increase in swelling ratio with an increase in the $\mathrm{pH}$ of the buffers. High $\mathrm{pH}$ value causes dissociation of carboxylic acid groups which strengthen the electrostatic repulsive force among anions of carboxylate and cause additional extension in polymers network consequently more swelling takes place [38].

In series AVD0.5-AVD2 the lowest swelling capacity is shown by AVD2 having a maximum amount of drug which showed a decrease in swelling behaviour with an increase in drug as we increase the amount of drug. It raised the cross-linked compactness of the polymer network and lessens free volume resulting decrease in water diffusion. Similar results are seen in the literature [40-42]. 

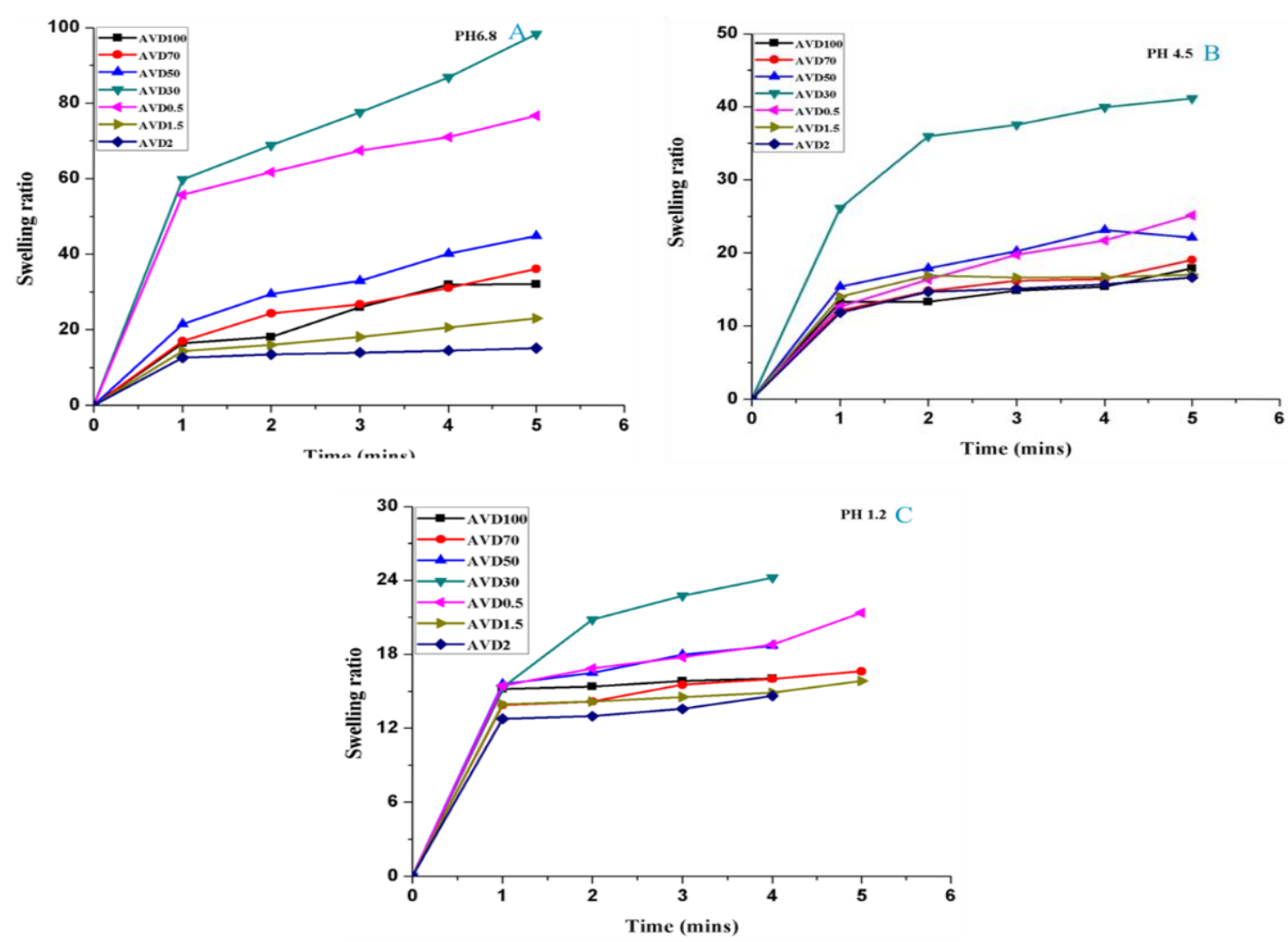

Fig. 9. Swelling ratio of drug loaded Sodium alginate/PVP/Claynanocomposite films in (A) Phosphate- pH 6.8 buffer solutions (B) NaOAc-pH 4.5 and $(\mathrm{C}) \mathrm{HCl}-\mathrm{pH} 1.2$ buffer solutions.

\subsubsection{Erosion Study}

All the formulations of clay reinforced polymer nanocomposites were studied for erosion behaviour in buffers of $\mathrm{pH} \mathrm{1.2,4.5}$ and 6.8. Table 1, inferred that formulation having higher concentration of PVP showed more erosion relative to formulation containing more SA. In series AV100-AV30, the AV30 showed maximum value of swelling $(90.4 \pm 0.001)$ in buffer solution of pH 6.8. Similar erosion behaviour is shown by series AVD100-AVD30.In series AVC50AVC20,the formulation AVC20 containing higher amount of clay showed more erosion as compared to AVC50(6) with lower amount of clay. This behaviour is attributed to increase in number of pores and decrease in size of pores upon addition of clay and this produces more channels for release of water which consequence a boost in de-swelling [23]. In series AVD 0.5AVD2, the formulation containing higher amount of drug showed more erosion as compared to formulation with lower concentration of drug. Similar behaviour is seen in buffer of pH 1.2 and 4.5.the erosion behaviour was higher in phosphate buffer of $\mathrm{pH} 6.8$ as compared to $\mathrm{PH} 4.5$ and 1.2. 
Table 1. Erosion Data for Sodium alginate/Polyvinyl pyrollidone formulations.

\begin{tabular}{|c|c|c|c|}
\hline \multirow{2}{*}{ Formulation codes } & \multicolumn{3}{|c|}{ Erosion $(\% \pm \mathrm{SD})$} \\
\hline & pH 1.2 & pH 4.5 & pH 6.8 \\
\hline AV100 & $64.7 \pm 0.000$ & $69.8 \pm 0.004$ & $71.1 \pm 0.004$ \\
\hline AV70 & $70.2 \pm 0.001$ & $82.4 \pm 0.003$ & $85.2 \pm 0.007$ \\
\hline AV50 & $80.9 \pm 0.004$ & $86.6 \pm 0.016$ & $88.4 \pm 0.002$ \\
\hline AV30 & $82.4 \pm 0.008$ & $83.7 \pm 0.000$ & $90.4 \pm 0.001$ \\
\hline AVD100 & $56.7 \pm 0.000$ & $57.2 \pm 0.018$ & $61.7 \pm 0.001$ \\
\hline AVD70 & $66.1 \pm 0.001$ & $71.7 \pm 0.002$ & $79.7 \pm 0.002$ \\
\hline AVD50 & $69.5 \pm 0.000$ & $72.4 \pm 0.004$ & $80.7 \pm 0.008$ \\
\hline AVD30 & $67.7 \pm 0.003$ & $81.4 \pm 0.005$ & $83.0 \pm 0.001$ \\
\hline $\operatorname{AVC50}$ & $54.5 \pm 0.014$ & $60.2 \pm 0.002$ & $71.1 \pm 0.018$ \\
\hline AVC10 & $55.3 \pm 0.025$ & $67.8 \pm 0.004$ & $74.2 \pm 0.000$ \\
\hline AVC20 & $61.0 \pm 0.02$ & $69.9 \pm 0.018$ & $76.7 \pm 0.020$ \\
\hline AVD0.5 & $44.7 \pm 0.007$ & $46.7 \pm 0.002$ & $66.9 \pm 0.003$ \\
\hline AVD1.5 & $54.3 \pm 0.020$ & $61.8 \pm 0.004$ & $69.5 \pm 0.021$ \\
\hline AVD2 & $63.9 \pm 0.002$ & $76.4 \pm 0.021$ & $79.8 \pm 0.005$ \\
\hline
\end{tabular}

\subsubsection{Calibration Curve of Ceftriaxone}

The serial dilution method was used to plot the standard calibration curve. Phosphate buffer of pH6.8 was used to make dilution in the range of $2-20 \mu \mathrm{g} / \mathrm{ml}$. The coefficient of determination $\left(R^{2}\right)$ value calculated was 0.996 and the value of Y-equation emerged to be $0.005 x+$ 0.135 


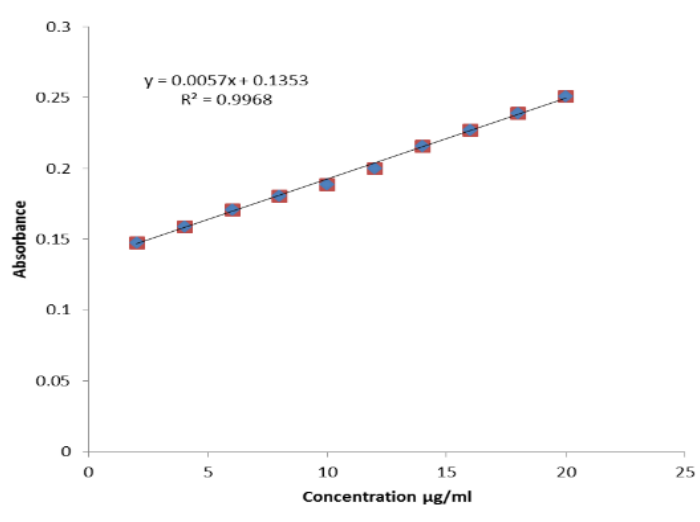

Fig. 10. Ceftriaxone Calibration Curve.

\subsubsection{Permeation study}

Permeation studies were carried out to calculate approximate rate of drug discharge from rat skin. The experiment was executed in triplicate with time break of 1, 2, 3, 4, 5, 6 and 24 hours in phosphate buffer solution of PH6.8 in Franz diffusion cell. The seriesAVD100-AVD30 the consequences of varying polymer with constant drug concentration on permeation via rat sin were analyzed in detail. The Figure 11 revealed the \%permeated through polymer nanocomposites films. This series contain 15\% drug while AVD0.5, AVD1.5 and AVD2 have 10\%, 30\% and 40\% drug. The results showed that as the concentration of drug is increased the \% permeated is also increased. Moreover the polymer blend of Sodium alginate and PVP with ratio of 70/30 in AVD70 and 30/70 in AVD30 showed maximum drug release in this series due to hydrophilic nature of PVP and Sodium alginate [23, 43]

\subsubsection{Dissolution study}

The dissolution study was carried out in triplicate using phosphate buffer of PH 6.8 and the \% permeated of drug discharge was estimated with time break of 1, 2, 3, 4, 5, 6 and 7 minutes. The drug discharge increase with increase in drug concentration in AVD2,AVD1.5,AVD0.5 while in series AVD100-AVD30 the maximum drug release was shown by AVD50 having sodium alginate and PVP ratio 50/50 having drug concentration 15\%. Increase in PVP concentration also enhanced the drug discharge [35].
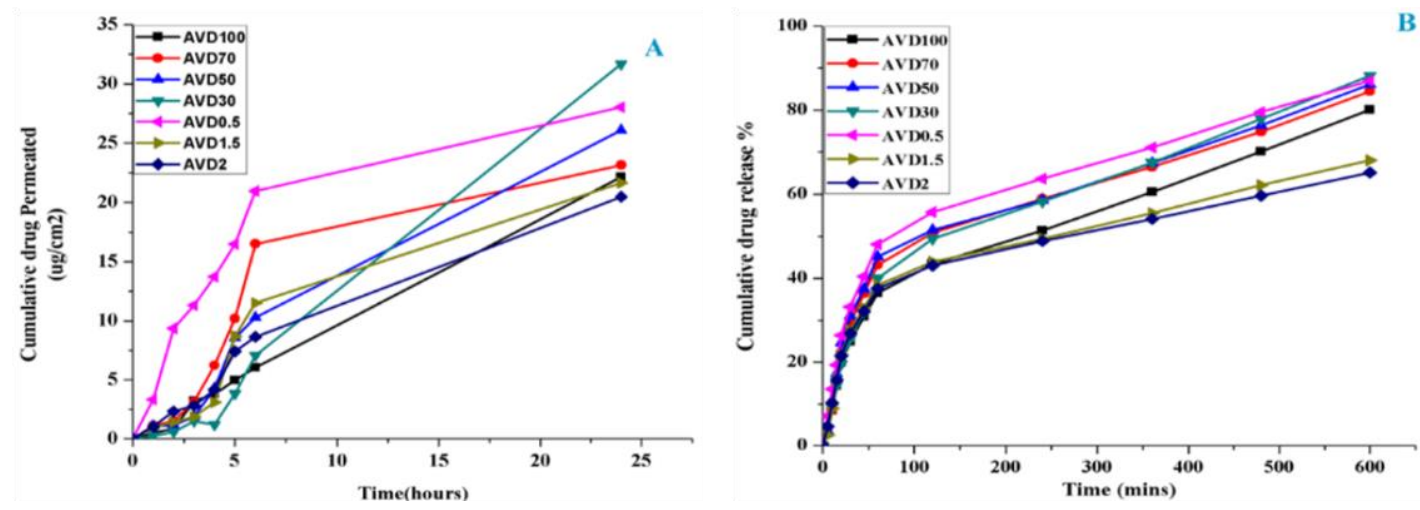

Fig. 11. (A): Ceftriaxone permeation Comparison for Formulations APD100-APD2 (B): Ceftriaxone in vitro release Comparison for Formulation APD100-APD2.

\section{Conclusions}

In this exertion, biopolymer nanocomposite films were prepared by blending sodium alginate with PVP by solvent casting method using clay as reinforcement. Ceftriaxone sodium 
drug was loaded into sodium alginate/PVP matrix with high percentage of alliance effectiveness. Films created were approximately white, flexible like plastic. The TGA data of formulations (Sodium alginate/PVP) reveals that sodium alginate's thermally stability increases with addition of PVP. Moreover, addition of nanoclay increased their thermal stability only to an optimum value; however, the addition of drug increases the thermal stability of drug loaded nanocomposites. XRD investigation manifests the semi-crystalline character of sodium alginate and PVP. Sharp peaks with reduced $2 \theta$ and higher d- spacing values were observed for nanoclay. The drug loaded formulations are amorphous in nature showed broad peaks. Scanning electron micrograph and EDX analysis revealed the uniform distribution and purity of mixing components. Swelling and erosion analysis were performed in buffers of $\mathrm{pH} \mathrm{1.2,4.5}$ and 6.8. The maximum swelling and erosion were observed in $6.8 \mathrm{pH}$. However, all those formulations having high concentration of PVP revealed the higher value of swelling and erosion. According to dissolution and permeation data, an increase in PVP concentration enhanced the drug permeability and dissolution.

\section{Acknowledgments}

The authors are grateful to COMSAT University Islamabad, Abbottabad campus and Govt. girl's degree college Qalandrabad, Abbottabad for the provision of laboratory facilities for research wok.

\section{References}

[1] Lorna Relleve, Fumio Yoshii, Alumanda dela Rosa, Tamikazu Kume, Angew. Makromol. Chem. 273, 63 (1999). https://doi.org/10.1002/(SICI)1522-9505(19991201)273:1<63::AIDAPMC63>3.0.CO;2-G

[2] C. S Fuller, R. J. MacRae, M. Walther, R. E. Cameron: Polym. Plast. Technol. Eng. 42, 9583 (2001). https://doi.org/10.1016/S0032-3861(01)00477-3

[3] M. Miya, R. Iwamoto, S. Mima, J. Polym. Sci.Part B Polym. Phys. 22, 1149 (1984). https://doi.org/10.1002/pol.1984.180220615

[4] Raymond Wai Man Ng, Yat Loong Cheng: J. Burn.Care Res. 28, 203 (2007). https://doi.org/10.1097/BCR.0B013E31802CA471

[5] Størker Moe, Gudmund Skjåk-Bræk, Olav Smidsrød, Hisao Ichijo : J. Appl. Polym. Sci. 51, 1771 (1994). https://doi.org/10.1002/app.1994.070511010

[6] John J. Meister. Poymer modification: principles, techniques, and applications, Marcel Dekker, Inc, New York, 203 (2000).

[7] M George, T E Abraham : Int. J. Pharmaceut. 335, 123 (2007).

https://doi.org/10.1016/j.ijpharm.2006.11.009

[8] Jeanie L Drury, David J Mooney, Biomaterials 24, 4337 (2003).

https://doi.org/10.1016/S0142-9612(03)00340-5

[9] Orive G. Ponce,S. Hernández, R. M. Gascón, A. R. Igartua, M. Pedraz, Biomaterials 23, 3825, (2002). https://doi.org/10.1016/S0142-9612(02)00118-7

[10] Guizhen Ke, Weilin Xu, Weidong Yu, Int .J. Polym. Mater. 59, 184 (2010). https://doi.org/10.1080/00914030903231332

[11] Olagoke Olabisi, Lloyd M. Robeson, Montgomery T. Shaw, J. Polym. Sci. Polymer Letters Edition 18, 582 (1980). https://doi.org/10.1002/pol.1980.130180815

[12] L. A. Utracki, Journal of Polymer Science Part C: Polymer Letters 28, 387 (1990).

https://doi.org/10.1002/polb.1990.090280310

[13] M. J. Folkes, P. S. Hope, Polymer Blends and Alloys 203, 423 (1993).

https://doi.org/10.1007/978-94-011-2162-0

[14] Z.-M. Liu, Z.-K. Xu, J.-Q. Wang, J. Wu, J.-J. Fu, Eur. Polym. J. 40, 2077 (2004).

https://doi.org/10.1016/j.eurpolymj.2004.05.020

[15] S. P. Jin, M. Z. Liu, S. L. Chen, C. M. Gao, Eur. Polym. J. 44, 2162 (2008).

https://doi.org/10.1016/j.eurpolymj.2008.04.017 
[16] Lynda Lamoudi, Jean Claude Chaumeil, Kamel Daoud, Drug Deliv. Sci. Technol. 31, 93 (2016). https://doi.org/10.1016/j.jddst.2015.12.005

[17] Natthaporn Limpan, Thummanoon Prodpran, Soottawat Benjakul, Surasit Prasarpran, Food Hydrocoll. 29, 226 (2012). https://doi.org/10.1016/j.foodhyd.2012.03.007

[18] Jie Xie , Wenna Meng, Deyi Wu, Zhenjia Zhang, Hainan Kong, Journal of Hazardous Materials 231-232, 57 (2012). https://doi.org/10.1016/j.jhazmat.2012.06.035

[19] Gholam Reza Mahdavinia, Hossein Etemadi, Mater. Sci. Eng. C Mater. Biol. Appl. 45, 250 (2014). https://doi.org/10.1016/j.msec.2014.09.023

[20] Sangamesh G. Kumbar, Kumaresh S. Soppimath, Tejraj M. Aminabhavi, J. Appl. Polym.Sci. 87, 1525 (2003). https://doi.org/10.1002/app.11552

[21] Yihong Huang, Jun Lu, Chaobo Xiao, Polymer Degradation and Stability 92, 1072 (2007). https://doi.org/10.1016/j.polymdegradstab.2007.02.011

[22] Tuncer Caykara, Serkan Demirci, Omer Kantoğlu, Polymer-Plastics Technology and Engineering 46, 737 (2007). https://doi.org/10.1080/03602550701273971

[23] Meltem Kasapoglu Calik, Murat Ozdemir, J. Appl. Polym. Sci. 133, 5 (2016).

[24] K. Kiran Kumar, M. Ravi, Y. Pavani, S. Bhavani, A. K. Sharma, V. V. R. Narasimha Rao, J. Membr. Sci. 454, 200 (2014). https://doi.org/10.1016/j.memsci.2013.12.022

[25] Xin-GuiLi, IngoKresse, JürgenSpringer, JörgNissen, Yu-Liang Yang, Polymer - Plastics Technology and Engineering 42, 6859 (2001). [26] Azam Rashidzadeh, Ali Olad, Carbohydr. Polym. 114, 269 (2014). https://doi.org/10.1016/j.carbpol.2014.08.010

[27] Faranak Dehghani, Nafiseh Farhadian, Shiva Golmohammadzadeh, Amir Biriaee, Mahmoud Ebrahimi, Mohammad Karimi, Eur. J. Pharm.Sci.96, 479 (2017).

https://doi.org/10.1016/j.ejps.2016.09.033

[28] Ali Olad, Hamed Gharekhani, Abdolreza Mirmohseni, Ahmad Bybordi, Advances in Polymer Technology 37, 1 (2017). https://doi.org/10.1016/j.carbpol.2017.03.047

[29] J. G. Jegal, K. H. Lee, J. Appl. Polym.Sci.61, 389 (1996).

https://doi.org/10.1002/app.1996.070610223

[30] K. Majdzadeh-Ardakani, B. Nazari: Compos. Sci. Technol. 70, 1557 (2010).

https://doi.org/10.1016/j.compscitech.2010.05.022

[31] Muralidharan Nagarajan , Soottawat Benjakul, Thummanoon Prodpran , Ponusa Songtipya, Int.J.Biol.Macromol. 75, 388 (2015). https://doi.org/10.1016/j.ijbiomac.2015.01.034

[32] Magdy W. Sabaa, Heba M. Abdallah, Nadia A. Mohamed, Riham R. Mohamed, Mater. Sci. Eng. C Mater. Biol. Appl. 56, 363 (2015). https://doi.org/10.1016/j.msec.2015.06.043

[33] Lifeng Qi , Zirong Xu, Xia Jiang, Caihong Hu, Xiangfei Zou: Carbohydr. Res. 339, 2693 (2004). https://doi.org/10.1016/j.carres.2004.09.007

[34] Jong-WhanRhim: LWT Food Sci.Technol. 37, 323 (2004).

https://doi.org/10.1016/j.lwt.2003.09.008

[35] Mustafa Yiğitoğlu, Gülden Aydın, Nuran Işıklan, Polym. Bull. 71, 385 (2014).

https://doi.org/10.1007/s00289-013-1067-0

[36] Ayelet Dar, Michal Shachar, Jonathan Leor, Smadar Cohen: Biotechnology and Bioengineering 80, 305 (2002). https://doi.org/10.1002/bit.10372

[37] Yuichi Ohya, Masahiro Shiratani, Hironao Kobayashi, Tatsuro Ouchi: Pure Appl. Chem. 31, 629 (1994). https://doi.org/10.1080/10601329408545334

[38] Y. M. A. C. Qin, X. D. Wang, K. Gilding, Alginate fibers and dressings. Text. Mag. 25, 22 (1996).

[39] Meltem Kasapoglu Calik, Murat Ozdemir, Appl. Polym. Sci. 133, 5 (2016).

[40] Sangamesh G. Kumbar, Tejraj M. Aminabhavi, Appl. Polym.Sci. 89, 2940 (2003).

https://doi.org/10.1002/app.12386

[41] Oya Sanli, Nuran Ay, Nuran Işiklan, Eur. J. Pharm. Biopharm. 65, 204 (2007).

[42] P. Manimekalai, R. Dhanalakshmi, R. Manavalan, Int. J. App. Pharm. 9, 10 (2017).

https://doi.org/10.22159/ijap.2017v9i6.16317

[43] Ebru Kondolot Solak, Oya Şanl1, Sep. Sci. Technol. 45, 1354 (2010).

https://doi.org/10.1080/01496391003608256 